\title{
Effect of Nitrogen Gas on the Lifetime of Carbon Nanotube Field Emitters for Electron-Impact Ionization Mass Spectrometry
}

\author{
Stephanie A. Getty*a ${ }^{\text {, }}$ Rachael Bis ${ }^{\mathrm{a}}$, Stacy Snyder ${ }^{\mathrm{b}}$, Emily Gehrels ${ }^{\mathrm{a}}$, Kristina Ramirez ${ }^{\mathrm{a}}$, Todd King ${ }^{\mathrm{a}}$, \\ Patrick Roman ${ }^{c}$, Paul Mahaffy ${ }^{\mathrm{d}}$ \\ ${ }^{a}$ NASA Goddard Space Flight Center, Materials Engineering Branch, Mailstop 541.0, Greenbelt, \\ MD 20771 \\ ${ }^{\mathrm{b}}$ Lehigh University, Physics Department, 16 Memorial Drive East, Bethlehem PA 18015 \\ 'NASA Goddard Space Flight Center, Detector Systems Branch, Mailstop 553.0, Greenbelt, MD \\ 20771 \\ ${ }^{d}$ NASA Goddard Space Flight Center, Atmospheric Experiments Laboratory, Mailstop 699.0, \\ Greenbelt, MD 20771
}

\begin{abstract}
The lifetime of a patterned carbon nanotube film is evaluated for use as the cold cathode field emission ionization source of a miniaturized mass spectrometer. Emitted current is measured as a function of time for varying partial pressures of nitrogen gas to explore the robustness and lifetime of carbon nanotube cathodes near the expected operational voltages $(70-100 \mathrm{eV})$ for efficient ionization in mass spectrometry. As expected, cathode lifetime scales inversely with partial pressure of nitrogen. Results are presented within the context of previous carbon nanotube investigations, and implications for planetary science mass spectrometry applications are discussed.
\end{abstract}

Keywords: Carbon nanotube, field emission, lifetesting, electron-impact ionization, sputtering

*Stephanie.A.Getty@nasa.gov; phone 1301 286-9760; fax 1301 286-1646

\section{INTRODUCTION}

Carbon nanotubes (CNTs) have been under evaluation as an alternative to Spindt emitters for cold cathode electron sources for over a decade [1-6]. While much research has demonstrated that CNTs are robust to some of the degradation mechanisms of Spindt emitters, such as runaway filamentary growth, the molecular nature of the CNT surface introduces additional degrees of instability. Typically, these instabilities are manifested as adsorbate-assisted field emission [7-10], chemical reactivity in the presence of oxidizing species $[7,8,11]$, and local melting and atomic rearrangement via Joule heating [12-14]. Herein, we focus on the application of multiwalled carbon nanotube emitters as an electron impact ionization source for a miniaturized mass spectrometer for use on planetary science missions throughout the solar system. In many of these environments, oxidizing species occur at low concentrations, compared to what is found on Earth, and therefore, this study explores the role of mechanical degradation by controlled leaks of nitrogen near the operational voltages of the CNT electron gun for mass spectrometry.

Mass spectrometers are a valuable tool for in situ chemical analysis of planetary atmospheres and surfaces in solar system exploration [15,16]. Given NASA's portfolio of upcoming mission targets, including Mars, the systems of Jupiter and Saturn, Venus, near-Earth asteroids, and comets, the ability to analyze a broad range of chemical constituents with high sensitivity and mass resolution will continue to be a highly desirable instrument payload capability. On long duration spaceflight missions, minimizing power consumption, mass, and footprint is of key importance. To this end, we are developing a CNT electron gun for use as the electron impact ionization source of a miniaturized time-of-flight mass spectrometer. The use of CNTs promises low-power field emission at sufficiently low extraction voltages of 70$100 \mathrm{eV}$ to promote efficient ionization of molecular species of interest.

Conventional electron impact ionization sources use a hot thermionic filament to produce an electron beam. As reported previously, CNT field emitters can produce a comparable electron current at two orders of magnitude lower power consumption [17]. Compared to other low-power emission approaches, such as metal- or semiconductor-based Spindt emitters, CNTs have been shown to be mechanically robust to sputtering [7], a major degradation mechanism of field 
emission tips. To date, however, most lifetesting has been conducted in ultra-high vacuum at high extraction voltages, leading to high ion accelerating voltages. Little work exists in the low voltage regime that is of primary relevance to mass spectrometry. To explore the low-voltage sputter-induced degradation, we have tested the lifetime of CNT field emitters at an extraction voltage of a few hundred volts in various partial pressures of nitrogen. We find, as expected, that higher operating pressure correlates with shorter cathode lifetime, but the durability of our cathodes in high vacuum and at low voltage compares favorably with previous studies in ultra-high vacuum and high voltage.

\subsection{Factors Affecting Carbon Nanotube Field Emission Stability and Lifetime}

Despite the considerable promise of using CNTs in field emission applications, a number of obstacles to implementation remain, including large fluctuations in emission current, thermal instability, and material evaporation in the high current regime. Previous work has shown that electromechanical rearrangement [18], a work function that depends on local CNT atomic structure [19], and adsorbed gas molecules can contribute to noisy emission current. Adsorption-enhanced emission has been seen in the presence of $\mathrm{H}_{2} \mathrm{O}$ [7,9], $\mathrm{N}_{2}$ [10], $\mathrm{CH}_{4}$, and $\mathrm{C}_{2} \mathrm{H}_{4}$ [20]. Reversible current degradation has been shown in the presence of $\mathrm{CO}$, and $\mathrm{CO}_{2}[20]$.

The lifetime of CNT emitters has been shown to outperform conventional Spindt emitters in robustness to mechanical sputtering and protrusion growth, owing to the small cross-section and the low sputter rate of carbon [7]. Due to the chemistry of the CNT structure, however, CNT emitters are susceptible to high partial pressures of specific types of oxidizing gases; $\mathrm{O}_{2}$ and $\mathrm{H}_{2} \mathrm{O}$ have been shown to irreversibly degrade CNT emitters through an oxidation process $[7,8]$.

To our knowledge, only a handful of publications have discussed direct measurements of CNT lifetime, including the seminal work in ultra-high vacuum by Dean and Chalamala. Although this published work does not specifically discuss the extraction voltage used to achieve emission, it can be approximated. Knowing that typical turn-on voltages of CNT emitters are above $1 \mathrm{~V} / \mu \mathrm{m}$, and given that the cathode-anode spacing in their diode structure is $5 \mathrm{~cm}$, we can estimate that the cathode-anode voltage was at least $50 \mathrm{kV}$. This is two to three orders of magnitude higher that the voltages used in mass spectrometry, and because this value determines the ion accelerating voltage during sputtering and reactive etching, the lifetime of a CNT field emitter should depend strongly on this parameter. Here, we discuss the lifetime of CNT emitter sources as a function of partial pressure of $\mathrm{N}_{2}$ in a low voltage regime.

\section{METHODOLOGY}

\subsection{Fabrication of Patterned Carbon Nanotube Field Emission Cathodes}

CNTs are grown using catalyst-assisted chemical vapor deposition. A silicon substrate is patterned using electron-beam lithography or photolithography, and a bilayer catalyst film of an aluminum underlayer and an iron top layer is thermally deposited in a high vacuum chamber, followed by conventional liftoff in acetone. Our current patterned cathode design features $5 \mu \mathrm{m} \times 5 \mu \mathrm{m}$ catalyst pads with a center-to-center spacing of $55 \mu \mathrm{m}$. Spacing is large compared to tower dimensions to produce an electrostatic profile that is favorable for field emission. The overall cathode pattern dimensions measure $2 \mathrm{~mm} \times 2 \mathrm{~mm}$, such that approximately 1500 individual towers comprise each cathode.

After catalyst deposition and patterning, the substrate is introduced into a chemical vapor deposition growth furnace and elevated to $750^{\circ} \mathrm{C}$ under flowing Ar. Following a $\mathrm{H}_{2}$ soak, ethylene is flowed over the catalyst to grow patterned CNT towers, as shown in a scanning electron micrograph in Figure 1.

As previously reported [17], CNT arrays of this design have been integrated into an electron gun with an extraction grid, an electrostatic lens stack, and an anode. The prototype electron gun exhibited Fowler-Nordheim tunneling behavior with an approximate field enhancement factor of 900 . 



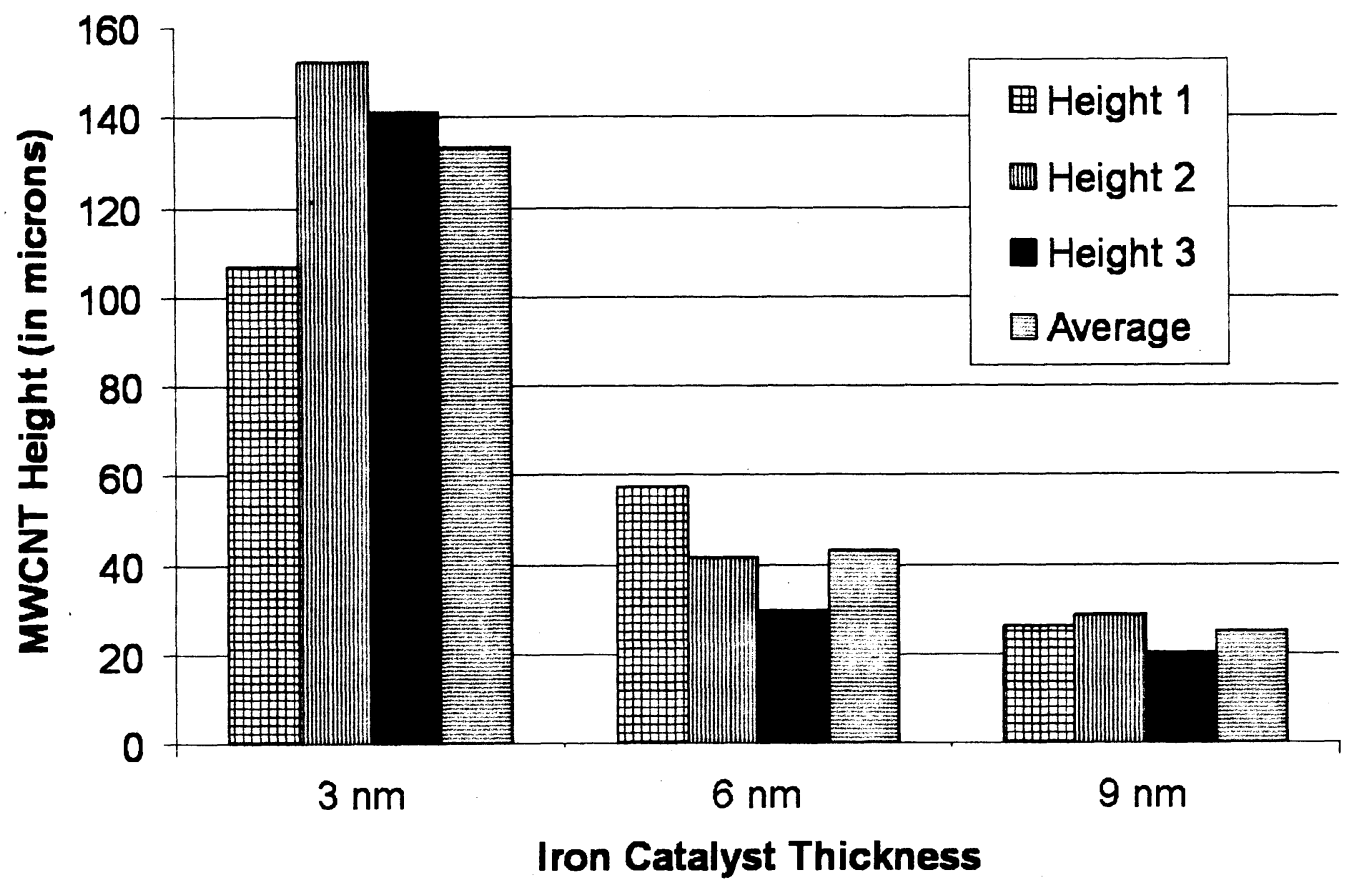

Fig. 2. CNT height shown as a function of iron catalyst thickness. An inverse relationship is seen, where the thickest catalyst produces the shortest CNTs.

\subsection{Fabrication of Silicon Extraction Grid}

Standard micromachining was used to fabricate a square-geometry silicon grid. Starting with a silicon-on-insulator wafer, photolithography and deep reactive ion etching were used to pattern square perforations in the device layer measuring $50 \mu \mathrm{m} \mathrm{x} 50 \mu \mathrm{m}$ with a bar width of $5 \mu \mathrm{m}$ to be commensurate with the dimensions of the cathode array. Deep reactive ion etching was then used to open a large aperture in the handle wafer, and buffered HF is used to open an aperture in the buried oxide. Voltage bias between this grid and the cathode produces the electric field for electron emission. An optical micrograph of a CNT patterned cathode-grid assembly, shown in Figure 3, reveals the defocused grid placed above the cathode towers. In this early prototype, the tower spacing is incommensurate with the grid perforations, and some towers are therefore obscured by the grid bars. Packaging designs currently under development include improvements such as micron-scale grid-cathode alignment. 


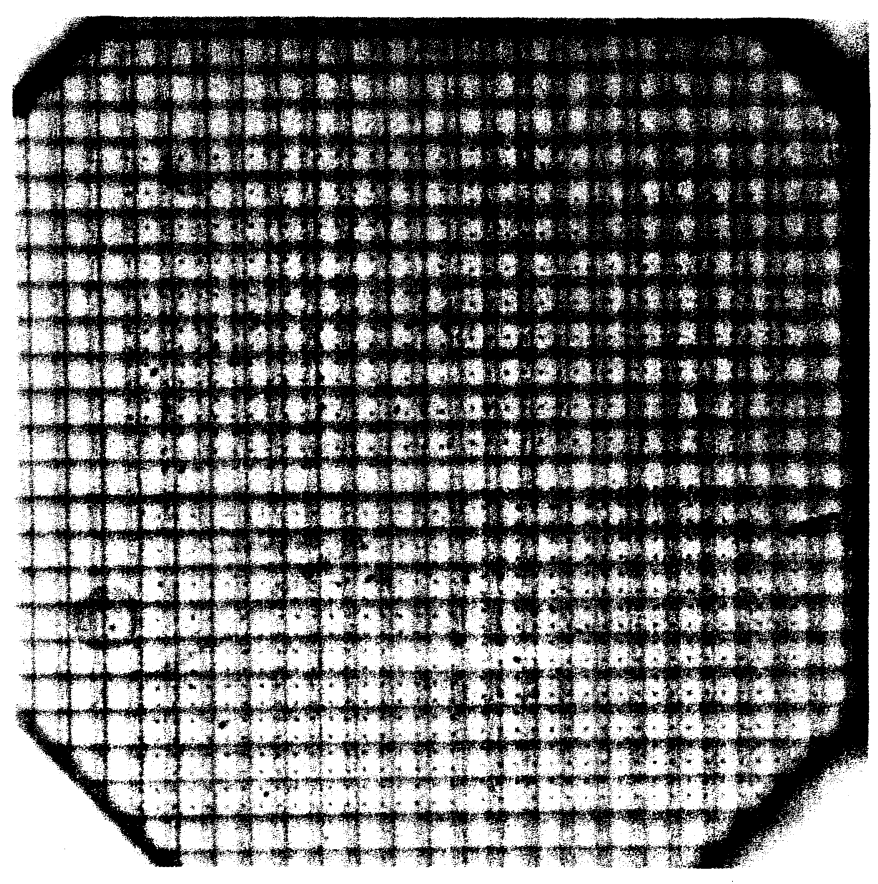

Fig. 3. Silicon grid is shown, defocused, above a CNT cathode. The tower array is barely visible as small dark points on a square lattice. Commensurate pitch and improved alignment will be implemented in future packaging approaches.

\subsection{Experimental Techniques}

Testing of our CNT field emission cathodes was performed in a high vacuum chamber with a nominal base pressure of $2 \times 10^{-7}$ torr. The chamber is fitted with a needle valve-modulated gas feedthrough for introducing controlled leaks into the chamber. The chamber features a residual gas analyzer (SRS RGA 300) for setting the leak gas partial pressure and for monitoring during testing.

The cathode is mounted inside a test fixture with integrated extraction grid, anode, and high voltage, vacuum-compatible wiring. The spacing between cathode and grid is determined by the height of the carbon nanotube towers, typically 10$20 \mu \mathrm{m}$, and the thickness of a glass spacer element that serves to electrically isolate the cathode and grid components. Here we use either a spacer with thickness of either $150 \mu \mathrm{m}$ (or $275 \mu \mathrm{m}$ in the case of cathode LP1) to give a cathodegrid spacing of approximately 130-140 $\mu \mathrm{m}$ (or 255-265 $\mu \mathrm{m}$ for LP1). For the lifetesting presented here, the spacers are affixed to the silicon frame of the cathode substrate using low-outgassing epoxy (Stycast 2850), but truly quantitative lifetime measurements will require the elimination of all epoxy. This is discussed further below.

To operate the CNT field emitter, the cathode is biased at negative voltages between 200 and $350 \mathrm{~V}$ using a high voltage source (SRS PS350). The current is measured at the grid by a dropping resistor method, where the collected current is sent across a dropping resistor to ground and the potential difference across the resistor is recorded. A LabView program allows computer control of the cathode bias voltage, and the current is collected at 1- to 30-second intervals for the duration of the lifetest. It should be emphasized that no ballast resistor or feedback control was used during the measurements.

\section{DATA}

Four cathodes were prepared as described above, and a separate lifetest was performed on each until failure. Two were lifetested at base vacuum, one at $\mathrm{N}_{2}$ partial pressure of $5 \times 10^{-6}$ torr, and one at $\mathrm{N}_{2}$ partial pressure of $1 \times 10^{-5}$ torr. The voltage is kept stable throughout the lifetest to isolate the effects of ion bombardment at the cathode tips; using this method, the kinetic energy of the sputtering ions is held constant. Due to differences in geometry and cathode-grid 
spacing, however, the operating voltage for each cathode varied between $200 \mathrm{~V}$ and $600 \mathrm{~V}$. Although these operating voltages are the same order of magnitude, we note that higher operating voltage is nevertheless expected to increase the energy of sputter ions and could contribute to measurement error. During setup, the voltage was increased until the current at the grid reached 5-10 $\mu \mathrm{A}$, and this determined the lifetest extraction voltage. For consistency, nominal completion of the lifetest is defined as the elapsed time at which the grid current approaches a stable, low-level flicker; the criterion for each cathode is discussed in further detail below. Reduced-performance lifetime is also presented, reflecting the persistence of diminished current for an extended duration. A summary of findings is shown in Figure 4.

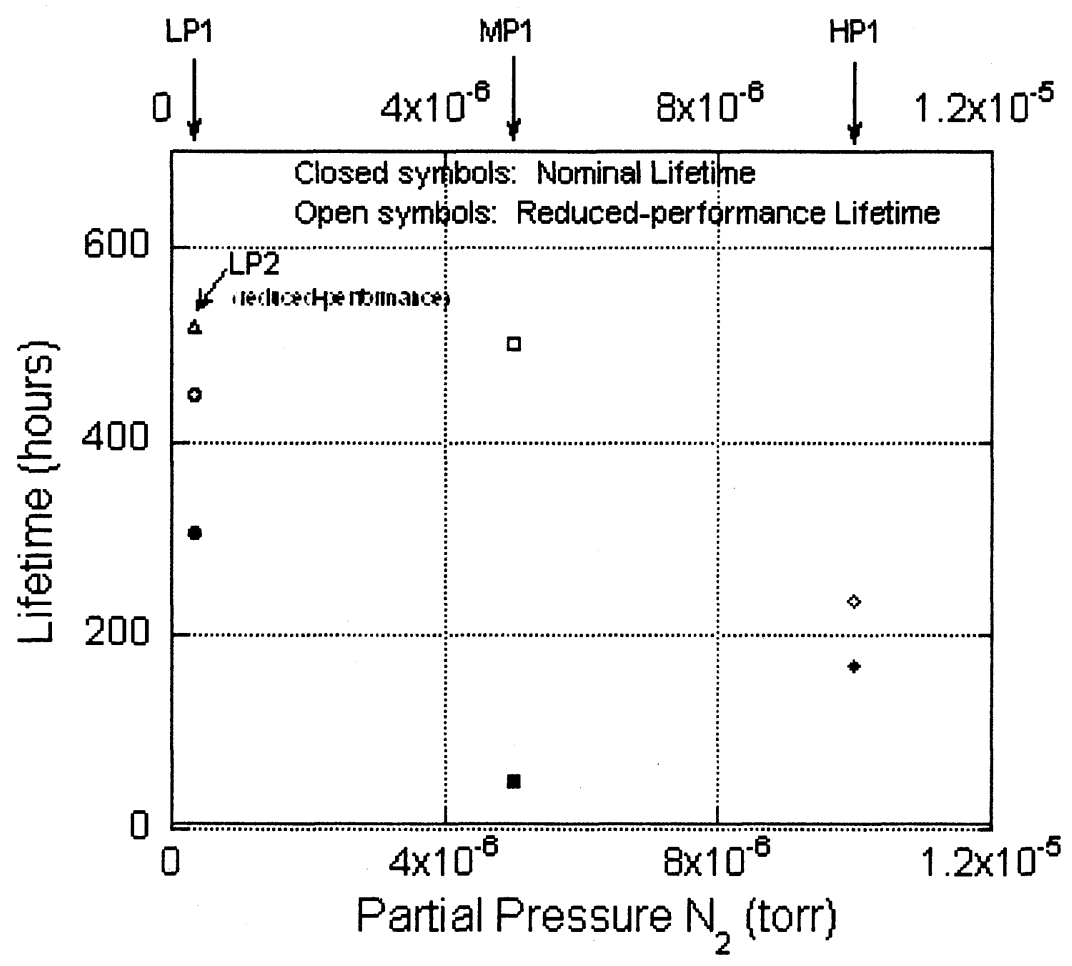

Fig. 4. Summary of lifetesting results as a function of partial pressure of $\mathrm{N}_{2}$ for cathodes LP1, LP2, MP1, and HP1. Nominal lifetime measurements are shown as solid symbols. Open symbols represent duration of diminished performance for each cathode.

\subsection{Base Pressure Lifetests}

Lifetests were performed independently on cathodes LP1 and LP2 at base vacuum as measured by the RGA, $3 \times 10^{-6}$ torr. Figure 5(a) shows grid current as a function of time for the duration of the lifetesting for LP1. Extraction voltage was relatively high at $600 \mathrm{~V}$, reflective of large cathode-grid spacing. Nevertheless, cathode LP1 operates at $\mu \mathrm{A}$ current levels without long-term degradation for approximately 300 hours. This is a lower bound estimate for the lifetime of LP1. While the current drops noticeably at 300 hours, the emission persists for at least 450 total hours. A second lowpressure lifetest was performed on cathode LP2 (data not shown), where detectable current persisted for at least 520 hours. 


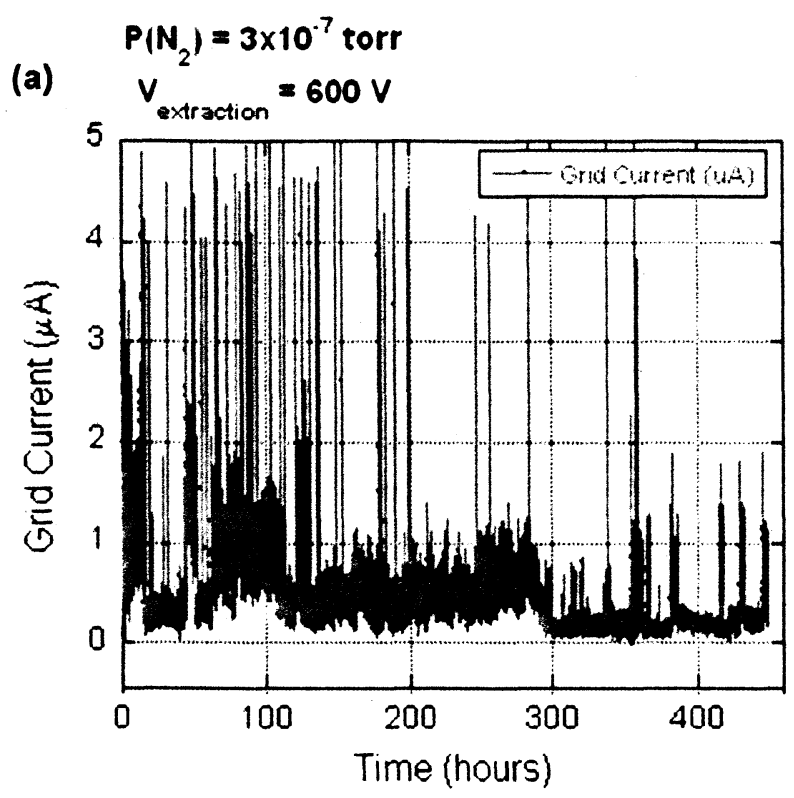

(b)

Sample LP1

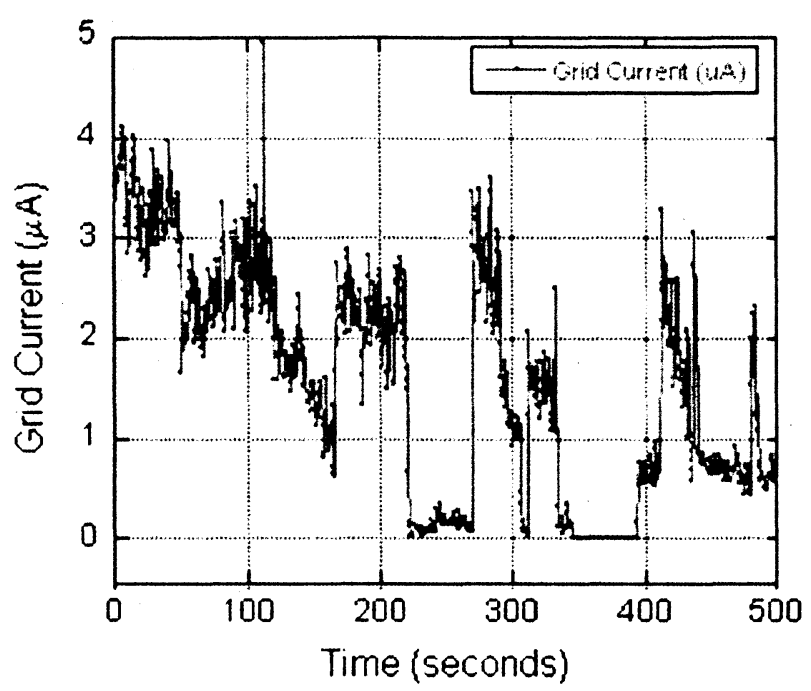

Fig. 5. Lifetesting results are shown for cathode LP1. (a) Current $(\mu \mathrm{A})$ versus elapsed time (seconds) for the duration of the lifetest. (b) High-resolution subset of lifetest data reveals time scales for signal noise.

A subset of the LP1 lifetesting data near the beginning of the lifetest is shown in Figure 5(b). In this set, data were collected at one-second intervals, revealing noise contributions from small high frequency fluctuations and low frequency telegraph noise. This low frequency switching is seen to diminish after approximately 15 minutes $(900$ seconds), and the high frequency switching dominates for the duration of the lifetest. The possible origin of each component is discussed further below.

\subsection{Intermediate Pressure Lifetest}

An intermediate-pressure lifetest is shown for cathode MP1 in Figure 6. To achieve an initial grid current of $10 \mu \mathrm{A}$, the extraction voltage was set to $200 \mathrm{~V}$. In Figure 6(a), current is shown as a function of time for operation of a CNT cathode in $5 \times 10^{-6}$ torr partial pressure of $N_{2}$. In the inset, we show the I-V characteristic for this device. As shown in the literature [11], hysteresis is seen between the up and down sweeps. The up sweep, taken at the beginning of the 24-hour baseline lifetest, is found to be noisier than the down sweep, recorded at the completion of the 24-hour baseline. This is consistent with reports that gaseous species are desorbed during operation. RGA scans taken at base pressure in the off state and emitting state are compared in Figure 7. Within the experimental sensitivity, significant differences in partial pressure are seen only for $\mathrm{H}_{2}$ and $\mathrm{N}_{2}$, two species known to be CNT adsorbates. We observe that the partial pressures of both gases increase during CNT cathode operation. This agrees with the expectation that gases are desorbed from CNT tips during emission. 


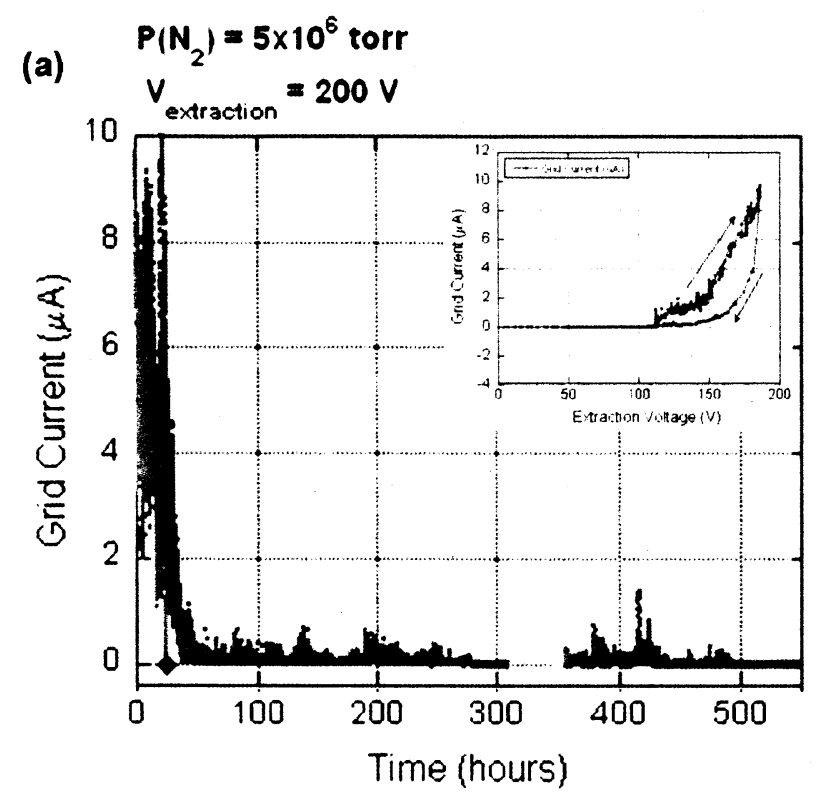

(b)

Sample MP1

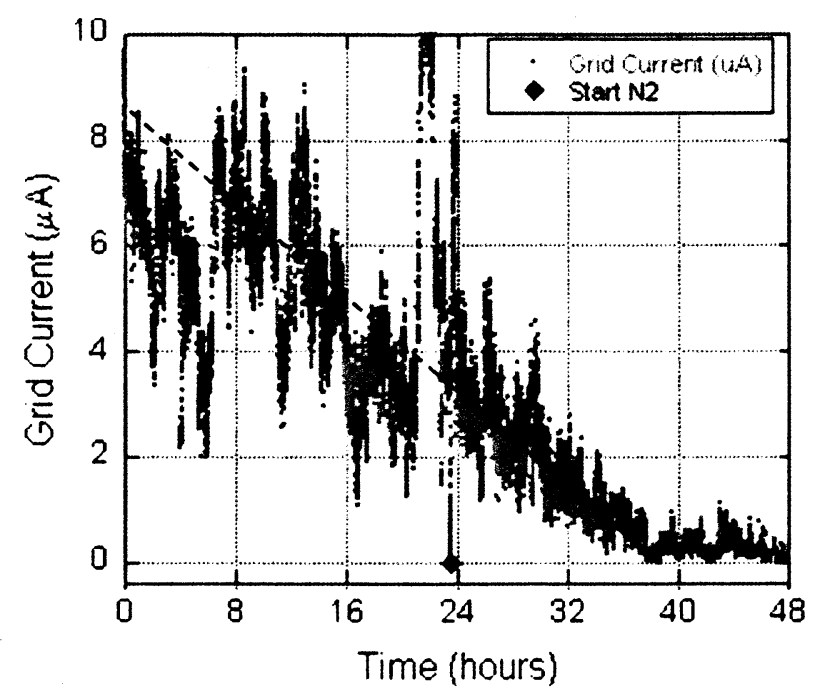

Fig. 6. Lifetesting results are shown for cathode MP1. (a) Current $(\mu \mathrm{A})$ versus elapsed time (seconds) for the duration of the lifetest. The blue diamond indicates the completion of the baseline lifetest and introduction of $\mathrm{N}_{2}$ into the chamber. Inset shows current-voltage characteristic. (b) Subset of lifetest data is shown for first 48 hours for comparison of behavior during baseline measurements and after introduction of $5 \times 10^{-6}$ torr partial pressure of $\mathrm{N}_{2}$.

Figure 6(b) shows the lifetest during the first two days. Upon the introduction of $\mathrm{N}_{2}$, indicated by a blue diamond, we can estimate the slope of the current degradation to be equal to or greater than that of the 24-hour baseline. A quantitative comparison is precluded by the level of signal noise. Near the end of the first 48 -hour period, the current stabilizes at a low level for the duration of the test. The 40-hour mark is considered the lower-bound lifetime of the MP1 cathode, but we emphasize that measurable current is recorded for at least 500 hours.

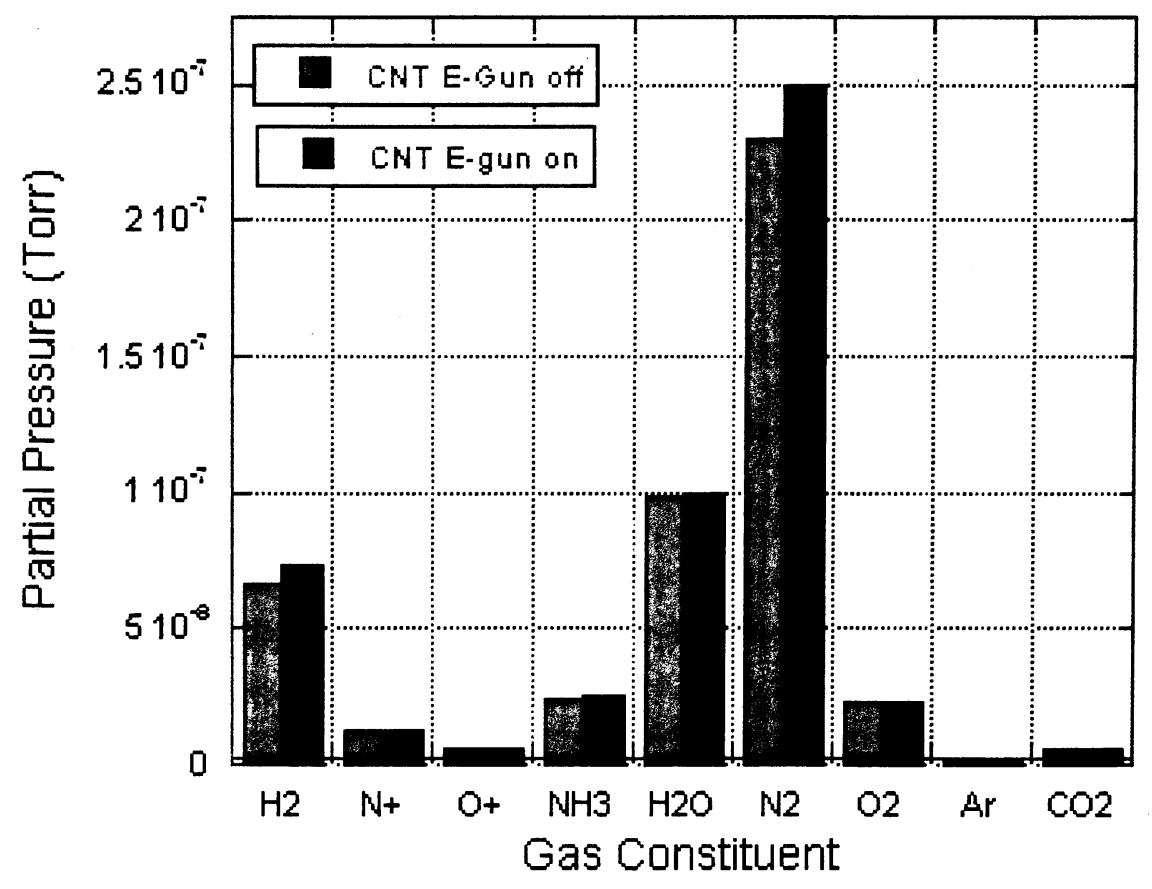


Fig. 7. RGA scans were taken while the CNT cathode was not emitting and during field emission. The partial pressures of $\mathrm{H}_{2}$ and $\mathrm{N}_{2}$, two known CNT adsorbates, increase during emitter operation.

\subsection{High Pressure Lifetest}

A high-pressure lifetest is shown for cathode HP1 in Figure 8. The entire lifetest is shown in Figure 8(a), where grid current is plotted as a function of time for a 24-hour baseline and subsequent operation in $1 \times 10^{-5}$ torr of $\mathrm{N}_{2}$. In the inset, an I-V characteristic is shown for this cathode. Here, the turn-on voltage is higher than in MP1, presumably due to slightly larger cathode-grid spacing, but the qualitative behavior is consistent between devices. The lifetest was conducted with an extraction voltage of $350 \mathrm{~V}$. Examining the first 48 hours of the lifetest, shown in Figure 8(b), it is difficult to conduct an analysis of the change in slope, as in the case of MP1, since the data are characterized by multiple slopes. A rapid degradation is observed shortly after introduction of $\mathrm{N}_{2}$, and this stable current level persists for approximately 170 hours, assigned as the lower bound for the lifetime of cathode HP1. The lifetest yields measurable current, however, for at least 235 total hours.

(a)

$$
P\left(N_{2}\right)=1 \times 10^{-5} \text { torr }
$$

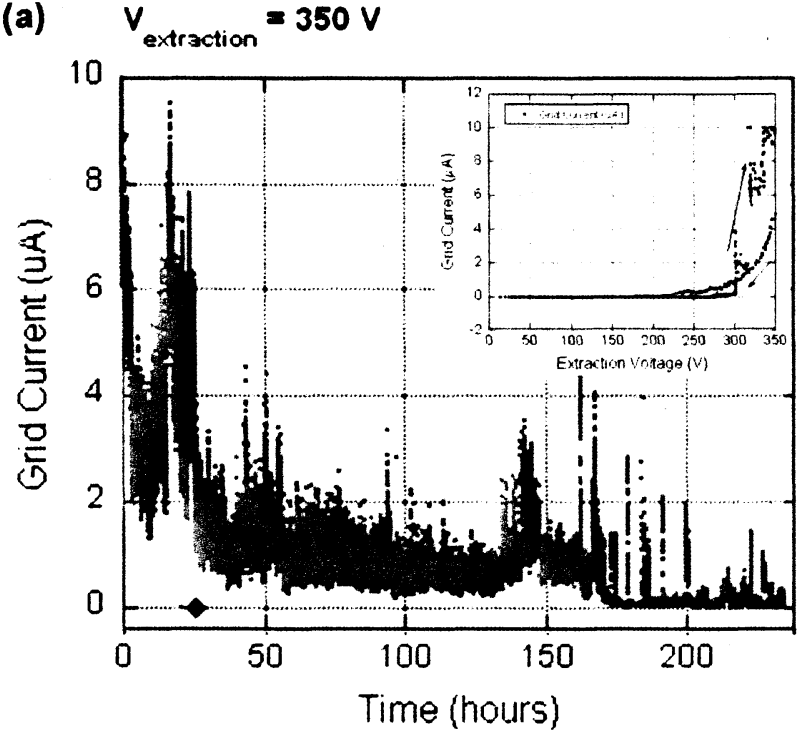

(b)

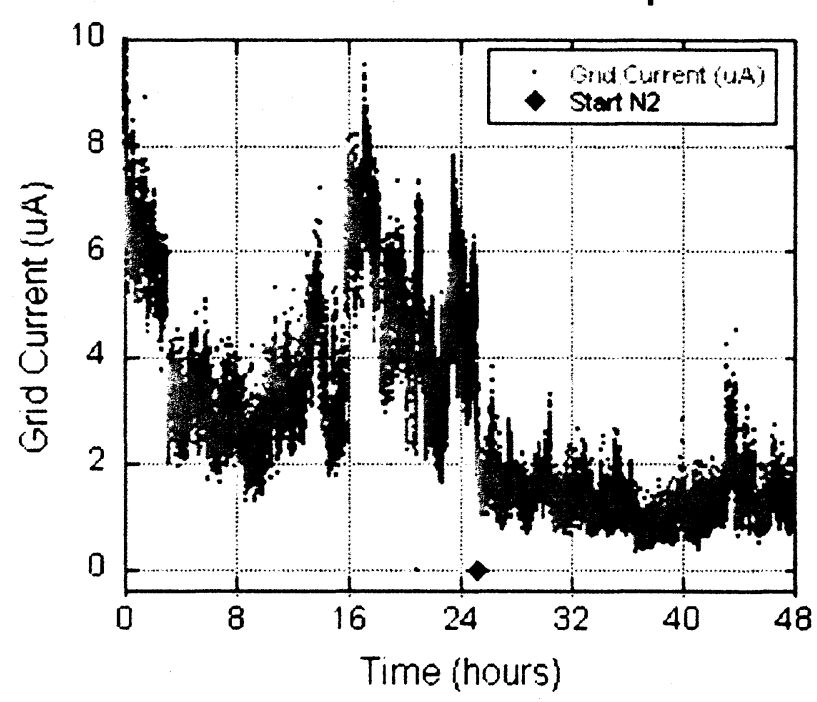

Fig. 8. Lifetesting results are shown for cathode HP1. (a) Current $(\mu \mathrm{A})$ versus elapsed time (seconds) for the duration of the lifetest. The blue diamond indicates the completion of the baseline lifetest and introduction of $\mathrm{N}_{2}$ into the chamber. Inset shows current-voltage characteristic. (b) Subset of lifetest data is shown for first 48 hours for comparison of behavior during baseline measurements and after introduction of $1 \times 10^{-5}$ torr partial pressure of $\mathrm{N}_{2}$.

\section{RESULTS}

We find that the base pressure lifetests consistently yielded longer lifetime in LP1 and LP2 than the elevated-pressure lifetests. The lifetests of emitters MP1 and HP1, however, do not reveal a clear trend in the lifetime dependence of CNT emitters on partial pressure of $\mathrm{N}_{2}$. The higher persistent current observed in HP1, compared to MP1, suggests the effect of adsorbate-enhanced tunneling, but further study is necessary to confirm this effect. Conclusions drawn from the body of data presented here might be reinforced by additional data sets at orders of magnitude higher pressure, but we conjecture that improved control and knowledge of the local pressure in the vicinity of the cathode is critical to fully quantifying the effects of sputtered gases on the lifetime of CNT emitters. 
We now turn to a discussion of the signal noise under the various vacuum conditions presented here. Two possible contributions to the current fluctuations in a CNT emitter are (1) emission through unstable adsorbate states and (2) electromechanical rearrangement of individual CNTs, giving rise to fluctuations in geometry, field enhancement factor, and cathode-grid spacing of individual emitters. To resolve the relative contributions of each effect, we observe that the noise exhibited by HP1 is clearly larger than that observed in MP1. This finding suggests that the higher concentration of molecular adsorbates at higher pressure is a contributor to the signal noise, consistent with current fluctuations due to the presence of adsorbates on the CNT tips [7,10]. The alternative explanation of electromechanical rearrangement of individual CNTs, in contrast, is expected to show no dependence on ambient pressure. Furthermore, the fluctuations due to electromechanical effects have been shown theoretically to occur on a time scale of several seconds [18], which may correspond to the low-frequency switching observed at early times for LP1 (Figure 5(b)). The observed telegraph noise diminishes after 15 minutes of testing, consistent with the system reaching an electromechanical equilibrium. The subsecond switching observed in the case of LP1 resembles high-frequency two-state fluctuations seen in the case of $\mathrm{N}_{2}$ adsorbates [10], suggesting that both mechanisms of adsorbate-assisted tunneling and electromechanical effects play a role in the emission current level and the magnitude of signal noise. The high-frequency dynamics of field emission from our CNT arrays will be pursued in future work to quantitatively determine the intrinsic time scale for these fluctuations.

Large scatter is observed in the lifetesting data presented here. Cursory analysis of these results seems to indicate that moderate levels of $\mathrm{N}_{2}$ partial pressure create more sputter damage than high levels, which is in direct contradiction to expectations. One likely contributor to the error in lifetime measurements reported herein is the use of epoxy during cathode assembly. Although the type of epoxy used is considered a low-outgassing epoxy, we acknowledge that the cleanliness requirements of this type of study probably preclude the use of soft polymers. Future efforts include the development of epoxy-free packaging that will promote an ultra-clean, ultra-high vacuum-compatible environment in the vicinity of the field emitters. Quantitative results will be obtained in future work for a subset of the lifetests presented here, but the general findings of this work are expected to extrapolate to ultra-clean device performance.

It is notable that, even at elevated levels of contamination, the lifetime of our CNT emitters compares favorably to the 350 hours reported in previous investigations [7]. Despite local pressure that is at least three orders of magnitude higher than our predecessors, emission current from our CNT cathodes persists for several hundred hours. We attribute this high-pressure longevity to an operating voltage that is two orders of magnitude lower in this work. Lower operating voltages lead to less energetic ions, thereby expected to reduce the detrimental effects of sputtering by local gases. This supports the expectation that the lower operational voltages used in mass spectrometry will promote longer cathode lifetime.

\section{CONCLUSIONS}

We have explored the lifetime of unballasted CNT emitters in the low operating voltage regime at varying partial pressures of $\mathrm{N}_{2}$. Shorter lifetime is observed to correlate with higher pressure, as expected, and differences in signal noise are well described by fluctuations due to adsorbate-assisted tunneling. Electromechanical rearrangement of individual CNTs are also expected to contribute to current fluctuations, independent of operating pressure. We have identified areas for future improvement of emitter assembly techniques to enable higher fidelity measurements of lifetime; these include minimizing the use of polymer materials and perhaps integrating a local getter. Extrapolation of the trends reported here is expected to be valid for an ultra-clean emitter assembly. Even in a contaminated environment, however, our CNT cathodes exhibit lifetimes that are competitive with previous results in UHV, and this robustness is attributed to low kinetic energy of the sputtering ions. Noise characteristics are expected to improve with integration of a ballast resistor and electronic feedback control. CNT longevity under conditions similar to an electronimpact ionization source suggests that field emission from a CNT cathode is a good candidate for use in mass spectrometry.

This research was supported by the Goddard Space Flight Center Internal Research and Development (IRAD) Program and the NASA Astrobiology Program. S.G. would like to thank Barney Lynch, Brian Jamieson, and Lance Delzeit for numerous helpful discussions. S.G. also acknowledges contributions by Professor David Allred, Bryan Hicks, Jonathon Brame, and Johnathan Goodsell, participants in the 2006 and 2007 ESMD Faculty-Student Summer Internship Program, and Melissa Harrison, participant in the 2006 MUCERPI summer internship program. 


\section{REFERENCES}

[1] A. G. Rinzler, J. H. Hafner, P. Nikolaev, L. Lou, S. G. Kim, D. Tomanek, P. Nordlander, D. T. Colbert, and R. E. Smalley, Science 269, 1550 (1995).

[2] Yu. V. Gulyaev, L. A. Chernozatonskii, Ja. Kosakovskaja, N. I. Sinitsyn, G. V. Torgashov, and Yu. F. Zakharchenko, J. Vac. Sci. Technol. B 13, 435 (1995).

[3] W. A. de Heer, A. Chatelain, D. Ugarte, Science 270, 1179 (1995).

[4] P. G. Collins and A. Zettl, Appl. Phys. Lett. 69, 1969 (1996).

[5] Q. H. Wang, T. D. Corrigan, J. Y. Dai, R. P. H. Chang, and A. R. Krauss, Appl. Phys. Lett. 70, 3308 (1997).

[6] Bower, C. A., Gilchrist, K. H., Piascik, J. R., Stoner, B. R., Natarajan, S., Parker, C. B., Wolter, S. D., and Glass, J. T., "On-chip electron-impact ion source using carbon nanotube field emitters," Appl. Phys. Lett. 90, 124102 (2007).

[7] Dean, K. A. and Chalamala, B. R., "The environmental stability of field emission from single-walled carbon nanotubes," Appl. Phys. Lett. 75, 3017 (1999).

[8] Wadhawan, A., Stallcup II, R. E., Stephens II, K. F., Perez, J. M., and Akwani, I. A., "Effects of $\mathrm{O}_{2}$, Ar, and $\mathrm{H}_{2}$ gases on the field-emission properties of single-walled and multiwalled carbon nanotubes," Appl. Phys. Lett. 79, 1867 (2001).

[9] Dong, C. and Gupta, M. C., "Influences of the surface reactions on the field emission from multiwall carbon nanotubes," Appl. Phys. Lett. 83, 159 (2003).

[10] Waki, S., Hata, K., Sato, H, and Saito, Y., "Behaviors of single nitrogen molecule on pentagon at carbon nanotube tip observed by field emission microscopy," J. Vac. Sci. Technol. B 25, 517 (2007).

[11] Lim, S. C., Choi, Y. C., Jeong, H. J., Shin, Y. M., An, K. H., Bae, D. J., Lee, Y. H., Lee, N. S., and Kim, J. M., "Effect of gas exposure on field emission properties of carbon nanotube arrays," Adv. Mater. 13, 1563 (2001).

[12] Bonard, J.-M., Klinke, C., Dean, K. A., and Coll, B. F., "Degradation and failure of carbon nanotube field emitters," Phys. Rev. B 67, 115406 (2003).

[13] Doytcheva, M., Kaiser, M., and de Jonge, N., "In situ transmission electron microscopy investigation of the structural changes in carbon nanotubes during electron emission at high currents," Nanotechnology 17, 3226 (2006).

${ }^{[14]}$ Liang, X. H., Deng, S. Z., Xu, N. S., Chen, J., Huang, N. Y., and She, J. C., "Noncatastrophic and catastrophic vacuum breakdowns of carbon nanotube film under direct current conditions," J. Appl. Phys. 101, 063309 (2007).

[15] Niemann, H. H. B., Atreya, S. K., Bauer, S. J., Biemann, K., Block, B., Carignan, G. R., Donahue, T. M., Frost, R. L., Gautier, D., Haberman, J. A., Harpold, D., Hunten, D. M., Isreal, G., Lunine, J. I., Mauersberger, K., Owen, T. C., Raulin, F., Richards, J. E., Way, S.H., "The gas chromatograph mass spectrometer for the Huygens Probe," Space Science Reviews 104, 553 (2002).

[16] Mahaffy, P.R., "Exploration of the Habitability of Mars: Development of Analytical Protocols for Measurement of Organic Carbon on the 2009 Mars Science Laboratory," Space Science Reviews, DOI 10.1007/s11214-007-9223-1 (2007).

[17] Getty, S. A., King, T. T., Bis, R. A., Jones, H. H., Herrero, F., Lynch, B. A., Roman, P., and Mahaffy, P., "Performance of a Carbon Nanotube Field Emission Electron Gun," Proceedings of SPIE 6556, 18 (2007).

[18] Sinha, N., Mahapatra, D. R., Sun, Y., Yeow, J. T. W., Melnik, R. V. N., and Jaffray, D. A., "Electromechanical interactions in a carbon nanotube based thin film field emitting diode," Nanotechnology 19, 025701 (2005).

[19] Tan, C. M., Jia, J., Yu, W., "Temperature dependence of the field emission of multiwalled carbon nanotubes," Appl. Phys. Lett. 86, 263104 (2005).

[20] Sheng, L. M., Liu, P., Liu, Y. M., Qian, L., Huang, Y. S., Liu, L., and Fan, S. S., "Effects of carbon-containing gases on the field-emission current of multi-walled carbon nanotube arrays," J. Vac. Sci. Technol. A 21, 1202 (2003).

[21] Hata, K., Futaba, D. N., Mizuno K., Namai T., Yumura M., Iijima S., "Water-assisted highly efficient synthesis of inpurity-free single-walled carbon nanotubes,"Science 306, 1362 (2004). 